\title{
Curriculum for education and training of Medical Physicists in Nuclear Medicine ${ }^{\text {is }}$ Recommendations from the EANM Physics Committee, the EANM Dosimetry Committee and EFOMP
}

\author{
Alberto Del Guerra ${ }^{\mathrm{a}, *, 1}$, Manuel Bardies ${ }^{\mathrm{b}, 2}$, Nicola Belcari ${ }^{\mathrm{a}, 1}$, \\ Carmel J. Caruana ${ }^{\mathrm{c}, 3}$, Stelios Christofides ${ }^{\mathrm{d}, 3}$, Paola Erba ${ }^{\mathrm{e}}$, Cesare Gori ${ }^{\mathrm{f}, 3}$, \\ Michael Lassmann ${ }^{\mathrm{g}, 2}$, Markus Nowak Lonsdale ${ }^{\mathrm{h}, 1}$, Bernhard Sattler ${ }^{\mathrm{i}, 1}$, \\ Wendy Waddington ${ }^{\mathrm{j}, 1}$
}

\footnotetext{
a University of Pisa, Department of Physics, Largo B. Pontecorvo 3, I-56127 Pisa, Italy

${ }^{\mathrm{b}}$ UMR 1037 INSERM/UPS, Centre de Recherche en Cancérologie de Toulouse, Bât A3, 133 Route de Narbonne 31062 Toulouse cedex, France

c Biomedical Physics, Faculty of Health Sciences, University of Malta, Malta

${ }^{\mathrm{d}}$ Nicosia General Hospital, Medical Physics Department, Nicosia, Cyprus

e University of Pisa, Dipartimento Traslazionale e Nuove Tecnologie, Pisa, Italy

${ }^{f}$ Firenze University Hospital, Struttura Organizzativa Dipartimentale di Fisica Sanitaria, Firenze, Italy

' Universitaetsklinikum Wuerzburg, Klinik fuer Nuklearmedizin, Oberduerrbacher Str. 6, 97080 Wurzburg, Germany

${ }^{\mathrm{h}}$ Bispebjerg Hospital, Department of Clinical Physiology and Nuclear Medicine, Bispebjerg Bakke 23,

2400 Copenhagen, Denmark

' University Hospital Leipzig, Department for Nuclear Medicine, Liebigstrasse 18, 04103 Leipzig, Germany

${ }^{\mathrm{j}}$ Institute of Nuclear Medicine, UCL Hospitals, London NW1 2BU, UK
}

\footnotetext{
This paper is not subjected to peer review, being a joint EANM-EFOMP article. It is published under the sole responsibility of EFOMP.

* Corresponding author. Tel.: +39050 2214942; fax: +39050 2214333.

E-mail address: alberto.delguerra@df.unipi.it (A. Del Guerra).

1 EANM Physics Committee.

${ }^{2}$ EANM Dosimetry Committee.

${ }^{3}$ EFOMP.
} 


\section{KEYWORDS}

Medical Physics

profession;

Education and

training;

Physics in Nuclear

Medicine
Abstract Purpose: To provide a guideline curriculum covering theoretical and practical aspects of education and training for Medical Physicists in Nuclear Medicine within Europe. Material and methods: National training programmes of Medical Physics, Radiation Physics and Nuclear Medicine physics from a range of European countries and from North America were reviewed and elements of best practice identified. An independent panel of experts was used to achieve consensus regarding the content of the curriculum.

Results: Guidelines have been developed for the specialist theoretical knowledge and practical experience required to practice as a Medical Physicist in Nuclear Medicine in Europe. It is assumed that the precondition for the beginning of the training is a good initial degree in Medical Physics at master level (or equivalent). The Learning Outcomes are categorised using the Knowledge, Skill and Competence approach along the lines recommended by the European Qualifications Framework. The minimum level expected in each topic in the theoretical knowledge and practical experience sections is intended to bring trainees up to the requirements expected of a Medical Physicist entering the field of Nuclear Medicine.

Conclusions: This new joint EANM/EFOMP European guideline curriculum is a further step to harmonise specialist training of Medical Physicists in Nuclear Medicine within Europe. It provides a common framework for national Medical Physics societies to develop or benchmark their own curricula. The responsibility for the implementation and accreditation of these standards and guidelines resides within national training and regulatory bodies.

(c) 2012 Associazione Italiana di Fisica Medica. Published by Elsevier Ltd. All rights reserved.

\section{Introduction}

To assist clarity, in this document we will often refer to Medical Physicists working (or training for a career) in Nuclear Medicine as Nuclear Medicine physicists.

Nuclear Medicine is a branch or specialty of medicine and medical imaging that uses radionuclides (unsealed sources of ionising radiation) and relies both on the emission of different kinds of ionising radiation and on the process of radioactive decay for the diagnosis and treatment of disease. Nuclear Medicine physicists are members of the multi-disciplinary clinical team responsible for diagnosis and therapy of disease. The role of Nuclear Medicine physicists is to provide critical scientific input on the physical processes and technology that underpin the whole Nuclear Medicine pathway. In Nuclear Medicine procedures, radionuclides are combined with other chemical compounds or pharmaceuticals, to form radiopharmaceuticals. These radiopharmaceuticals, once administered to the patient, can localize to specific organs or cellular receptors. The external detection of radiation emitted by the radionuclide - often called a radiotracer - allows the behaviour of the compound within the body to be followed, thus providing relevant information on the metabolism of the vector, usually but not exclusively, through information obtained by imaging. This property of radiopharmaceuticals allows Nuclear Medicine the ability to determine the extent of physiological processes in the body, based on cellular function and physiology. Additionally, specific vectors combined with short-range particle emitters can by used to selectively irradiate targets (molecular radiotherapy). In this respect, Nuclear Medicine qualifies as an independent medical discipline providing "functional imaging" modalities and radiation oncology options in a clinical setting.

Generally, Nuclear Medicine physicists design and develop the framework of radionuclide handling and delivery, radiopharmaceutical dosimetry, optimisation, quality assurance of individual patient's treatments and of the medical imaging equipment, including radiation safety of the patient and staff. Specifically, the Nuclear Medicine physicist provides expert advice on the development, implementation and improvement of medical imaging with radionuclides and the treatment of benign or malignant diseases with radiopharmaceuticals. The Nuclear Medicine physicist's role is key to the enabling of the practice of safe, state-of-the-art Nuclear Medicine diagnosis and treatment.

In order to acquire and maintain sufficient knowledge and an appropriate level of competence, both initial and continuing education and training are necessary. European legislation has challenged many professional organisations to propose harmonised professional standards of high quality. The European Union's Directives concerning basic safety standards [1] and medical exposures [2] have made it a statutory requirement to involve physicists in the medical uses of ionising radiation and have given impetus to the discussions of education and training requirements in Medical Physics. The European Federation of Organisations for Medical Physics (EFOMP) and the European Association of Nuclear Medicine (EANM) have recently started collaborating on scientific, education and training issues of common interest in the field of Nuclear Medicine. The two organisations have a long-standing commitment to improve clinical practice, science, development, education and training. These guidelines represent the first common attempt by the two organisations to accommodate the contemporary requirements for the knowledge/skills/ competences needs in this rapidly evolving field.

\section{The role of EANM}

The European Association of Nuclear Medicine was founded in 1985 as the umbrella organisation of Nuclear Medicine in Europe and represents the sector towards the European 
Institutions. Within this role, the EANM aims at advancing science and education in Nuclear Medicine for the benefit of public health. Moreover, the EANM aims at promoting and coordinating, throughout Europe and beyond, discussion and exchange of ideas and results relating to the diagnosis, treatment, research and prevention of diseases through the use of unsealed radioactive substances. The goal of the EANM is thus to provide a suitable medium for the dissemination and discussion of the latest results in the field of Nuclear Medicine and related subjects.

The Physics Committee together with the Dosimetry Committee of EANM have the task to lead the formal input of Medical Physics advice to the EANM Executive Committee, and to coordinate the activities related to Medical Physics and Dosimetry within the EANM. The committees identify areas of Nuclear Medicine practice in Europe that would benefit from formal input from Medical Physicists, by way of guidelines, standards, education and training or evaluation. The Physics Committee in particular has established formal links with EFOMP in order to coordinate work on Physics and Instrumentation topics. The Dosimetry Committee collaborates with international organisations or committees, such as the MIRD committee of the American Society of Nuclear Medicine (SNM) and the IAEA (International Atomic Energy Agency). Both committees issue guidelines, published in the European Journal of Nuclear Medicine and Molecular Imaging (EJNMMI) and other European Journals in this field, on various topics related to Medical Physics in Nuclear Medicine. In addition, they participate in the elaboration of clinical guidelines by reviewing their Medical Physics content (image acquisition and processing, instrumentation QA, radiation safety and dosimetry).

\section{The role of EFOMP}

EFOMP is an umbrella organisation for national Medical Physics organisations, with one of its main objectives the harmonisation and promotion of the best practice of Medical Physics within Europe. To accomplish its goals, EFOMP has presented various recommendations and guidelines in a number of Policy Statements, which have been unanimously adopted by EFOMP Member Organisations. Policy Statement No. 9, "Radiation Protection of the Patient in Europe: The Training of the Medical Physics Expert in Radiation Physics or Radiation Technology" [3], is the EFOMP response to the Medical Exposure Directive, 97/ 43/Euratom [2]. Here EFOMP presents its recommendations on the role and the competence requirements of the Medical Physics Expert (MPE), as defined in this Directive [2], together with recommendations on education, training and Continuing Professional Development (CPD). General criteria for structured CPD have been laid down by EFOMP in Policy Statement No. 8, "Continuing Professional Development for the Medical Physicist" [4]. CPD is the planned acquisition of knowledge, experience and skills, both technical and personal, required for professional practice throughout one's working life. EFOMP recommends that all Medical Physicists who have completed their basic education and training should be actively involved in CPD to maintain and increase competence and expertise after qualification. The EFOMP approach to achieve harmonisation is to encourage the establishment of national education and training schemes at all levels in line with EFOMP recommendations. Guidelines for formal EFOMP recognition of National Registration Schemes for Medical Physicists were established in 1995 [5]. EFOMP approval requires inter alia clear statements of theoretical and practical competences, as well as training programmes consistent with the EFOMP policy on training, and a regular renewal mechanism. CPD is now being recommended as the best way to meet the requirement for a renewal mechanism, and Policy Statement No. 10 "Recommended Guidelines on National Schemes for Continuing Professional Development of Medical Physicists" [6], and Policy Statement No. 12 "The present status of Medical Physics Education and Training in Europe. New perspectives and EFOMP recommendations" [7], recommend National Member Organisations to set up their own detailed CPD Scheme. In Policy Statement No. 12 additional recommendations on education and training of Medical Physicists within the context of the current developments in the European Higher Education Area arising from "The Bologna Declaration", and with a view to facilitate the free movement of professionals within Europe, are given. Other initiatives in educational issues have been developed by EFOMP in cooperation with ESTRO [8] ("Guidelines for education and training of Medical Physicists in radiotherapy. Recommendations from an ESTRO/EFOMP working group."). EFOMP is a partner in the EC project to develop a guidance document on Medical Physics Expert (MPE) that includes the required Education and Training of the MPE. A more recent EC project is the updating of the "Guidelines on education and training in radiation protection for medical exposures" [9]. EFOMP is also a partner in this project.

This curriculum for Medical Physicists in Nuclear Medicine arises from the necessity to update the education and training requirements to accommodate today's competence needs in modern Nuclear Medicine.

This document includes also other competence areas as organizational competence, professionalism, communication, collaboration, social actions, in addition to Nuclear Medicine physics skills and knowledge.

\section{Definitions}

\section{Medical Physics and Medical Physicist (MP)}

There is a wide acceptance of the definition of Medical Physics. Internationally respected organizations such as IOMP (International Organization for Medical Physics), AAPM (American Association of Physicists in Medicine) and EFOMP have adopted similar definitions: "Medical Physics is an applied branch of physics concerned with the application of the concepts and methods of physics to the diagnosis and treatment of human disease".

In an IOMP Policy Statement still under preparation [10] an updated definition is given of Medical Physics and of the Medical Physicist:

"Medical Physics is a branch of Applied Physics, pursued by Medical Physicists, which uses physics principles, methods and techniques in practice and research for the 
prevention, diagnosis and treatment of human diseases with a specific goal of improving human health and well-being. Medical Physics may further be classified into a number of sub-fields (specialties), including the following:

- Radiation Oncology Physics

- Medical Imaging Physics

- Nuclear Medicine Physics

- Medical Health Physics (Radiation Protection in Medicine)

- Non-ionizing Medical Radiation Physics

- Physiological Measurement"

"Medical Physicists are professionals with education and specialist training in the concepts and techniques of applying physics in medicine. Medical Physicists work in clinical, academic or research institutions. Medical Physicists working in the clinical environment are health professionals, with education and specialist training in the concepts and techniques of applying physics in medicine, competent to practice independently in one or more of the sub-fields (specialities) of Medical Physics."

Hence a Medical Physicist is a person, qualified with a university degree majoring in physics with specialized education and training in the concepts and techniques of applying physics to medicine and healthcare. In some countries the term 'Medical Physics' is often used as synonymous with 'clinical Radiation Physics' whilst in others the interpretation is much broader, with considerable overlap with biomedical (or clinical) engineering.

A recent definition from the IAEA is as follows and explicitly relates Medical Physics to radiation protection:

"A Medical Physicist is a healthcare professional who specialises in the application of physics in medicine and has the knowledge and responsibility for the radiation protection of patients, staff and the public".

However, more detailed definitions are given for the professional Medical Physicist, according to their degree of education and training by the professional organizations, in particular by EFOMP, as in its Policy Statement No. 7 [11] and No. 10 [6].

For a matter of clarity in the following we will refer to Medical Physicist (MP) in accordance to the most recent IOMP definition, as described above [10].

\section{Medical Physics Expert (MPE)}

The title "Medical Physics Expert" was introduced somewhat vaguely in 1997 in Council Directive 97/43/Euratom of 30 June 1997 [2] on health protection of individuals against the dangers of ionising radiation in relation to medical exposure ("the MED directive"). It had to be transposed into the national legislation in all European countries, defining the Medical Physics Expert in national legislation. However, due to the lack of clarity or a uniformity of criteria, the definition of the Medical Physics Expert is implemented differently across Europe.
A new directive, the Euratom Basic Safety Standards Directive (Draft Version 24 February 2010) [12] gives a new definition of the Expert. This directive will revoke both Council Directive 96/29/Euratom of 13 May 1996 [1], concerning basic safety standards for the protection of the health of workers and the general public against the dangers arising from ionising radiation ("the BSS") and the MED directive [2]. The new directive provides a clearer definition of the Medical Physics Expert:

"An individual having the knowledge, training and experience to act or give advice on matters relating to Radiation Physics applied to medical exposure, whose competence to act is recognized by the competent authorities".

In 2010, the European Commission launched a 2-year project on MPE to provide for improved implementation of the MED and to facilitate the harmonization of MPE among the Member States aiming at their cross-border mobility ("Guidelines on Medical Physics Expert Project", TREN/09/ NUCL/SI2.549828). The MPE project was assigned to a Consortium led by the Complutense University of Madrid (http://portal.ucm.es/web/medical-physics-expert-

project). As a final outcome of this project a "European Workshop on Medical Physics Expert" was held in Sevilla (Spain) on 9-10 May 2011 and the results of the workshop are available at the website http://portal.ucm.es/web/ medical-physics-expert-project.

The Medical Physics Expert is an important role of the Medical Physicist with a high level of expertise in one or more specialities of Medical Physics associated with the use of ionising radiation in medicine. This is particularly true for an MP in Nuclear Medicine, where most if not all of the activities are related to the use of ionizing radiation.

The content of this curriculum is aimed to bring trainees up to the level of competence to act and practice independently in the field of Nuclear Medicine.

Hence, in the following guidelines the competences, skills and knowledge needed for Medical Physicists in Nuclear Medicine will be described.

\section{Education and training scheme}

Article 7 of the MED directive [2] states that "Member States shall ensure that practitioners and other individuals mentioned in Articles 5(3) and 6(3) have adequate theoretical and practical training for the purpose of radiological practices, as well as relevant competence in radiation protection. For this purpose Member States shall ensure that appropriate curricula are established and shall recognize the corresponding diplomas, certificates or formal qualifications".

Also under paragraph 3 of Article 7 is stated that "Member States shall ensure that continuing education and training after qualification is provided and, in the special case of clinical use of new techniques, the organization of training related to these techniques and the relevant radiation protection requirements".

One of the individuals mentioned in the above articles, is the Medical Physics Expert (MPE) and particularly, paragraph 3 of Article 6 states "In radiotherapy practices a Medical Physics Expert shall be closely involved. In 
standardized therapeutic Nuclear Medicine practices and in diagnostic Nuclear Medicine practices, a Medical Physics Expert shall be available. For other radiological practices, a Medical Physics Expert shall be involved, as appropriate, for consultation on optimization including patient dosimetry and quality assurance including quality control, and also to give advice on matters relating to radiation protection concerning medical exposures, as required".

As stated earlier, the Medical Physics Expert is a key role of the Medical Physicist and therefore the MPE is very relevant to the recognition of the profession and to the free movement of the MP in Europe. Hence, this has a strong impact on education and training of MPs and of Nuclear Medicine physicists in particular. Very recently ISCO (The International Standard Classification of Occupations) has accepted the recommendation by the ILO (International Labour Organization) and has listed Medical Physics among the professions. The MP has been classified within the physics group, but with the special provision that Medical Physicists are also part of the "healthcare professions" group, when they operate in the clinical environment [13].

Furthermore, a recognition by the European Union including the ability to move and work freely in any Member State requires that the MP profession meets the criteria set by Directive 2005/36/EC of the European Parliament and of the Council of 7 September 2005 [14] on the recognition of professional qualifications. This includes maintaining competence through continuous professional development.

Due to the rapid advancement of medical equipment and procedures in the medical field there is an increasingly high dependence on educated professionals. The quality level of the Medical Physics Service provided is linked to the number of such highly educated professionals in relation to the number and complexity of the medical devices available in a specific health-care facility. In addition, modern Health Care Services are facing continuously growing demand for increased competence, specialization and cost effectiveness. The Medical Physics Service of a healthcare facility faces the same demands. Hence, Continuing Professional Development is vital for the MP profession to function in tandem with the pace of change occurring in medical practice. CPD promotes excellence within the profession and protects the professionals and the public against the potential dangers of ionizing radiation and suboptimal equipment.

There has been a continuous discussion in the scientific community on the education and training scheme necessary for a "modern" Medical Physicist. The obsolescence of a simple Master in Science degree has been recently discussed [15], and emphasis has been placed on the necessity that research should be an integral part of a Medical Physics graduate program [16].

The education and training of the MP must be in line with the Bologna declaration, the tuning process for the educational structures in Europe and the recommendation of the European Parliament and of the council of 23 April 2008 on the establishment of the European qualifications framework for lifelong learning.

In particular, Learning Outcomes (LO) of educational and training programmes are expressed in terms of Knowledge, Skills and Competence (KSC): a) Knowledge covering facts, principles, theories, practices

b) Skills as the ability to use knowledge and know-how to complete tasks and solve problems (both cognitive skills involving the use of logical, intuitive and creative thinking and practical skills involving manual dexterity and the use of methods, materials, tools and instruments)

c) Competence meaning responsibility and autonomy.

LOs for the Medical Physicist (MP) are classified into two categories: Generic and Subject Specific.

Generic LO consist of skills, which are transferable across professions. They can be further classified into three sub-categories:

i) Instrumental skills: cognitive, methodological, technological, linguistic

ii) Interpersonal skills: individual abilities involving social and co operational aspects

iii) Systemic skills: involving combinations of understanding, sensibility and knowledge and usually involving prior acquisition of instrumental and interpersonal skills.

These generic LO are common to all academic professions and will not be discussed here.

Subject specific LO are those that are specific to the profession. These may be further classified into subcategories as determined by the particular profession (see Fig. 1). In the case of Subject Specific LO for the MP the following categories are proposed:

- LO appropriate for the MP as physical scientist (these are fundamental physics knowledge and measurement skills expected of all physicists)

- LO for the MP as a healthcare professional

- LO for the MP as clinical medical device/physical agents expert (these are common LO applicable to all medical devices and safety from all associated physical agents). This section could be further divided in subsections

- LO for the various specializations of the MP. These would essentially consist in the application of the LO in a) - c) above to the various specializations of the MP.

In the case of Medical Physicists, the areas of specializations are: Diagnostic \& Interventional Radiology, Nuclear Medicine, Radiotherapy, Non-ionizing Radiation Applications and Physiological Measurements. These guidelines will only consider those required for Nuclear Medicine and will be presented in the next section.

\section{Core curriculum for a Medical Physicist in Nuclear Medicine}

In the following three Subsections the KSC as required for a Medical Physicist and common to all specialties are indicated. These are a prerequisite before developing the necessary KSC for any subspecialty area of Medical Physics. In the last Subsection the KSC specifically needed for a Nuclear Medicine Physicist will be illustrated. 


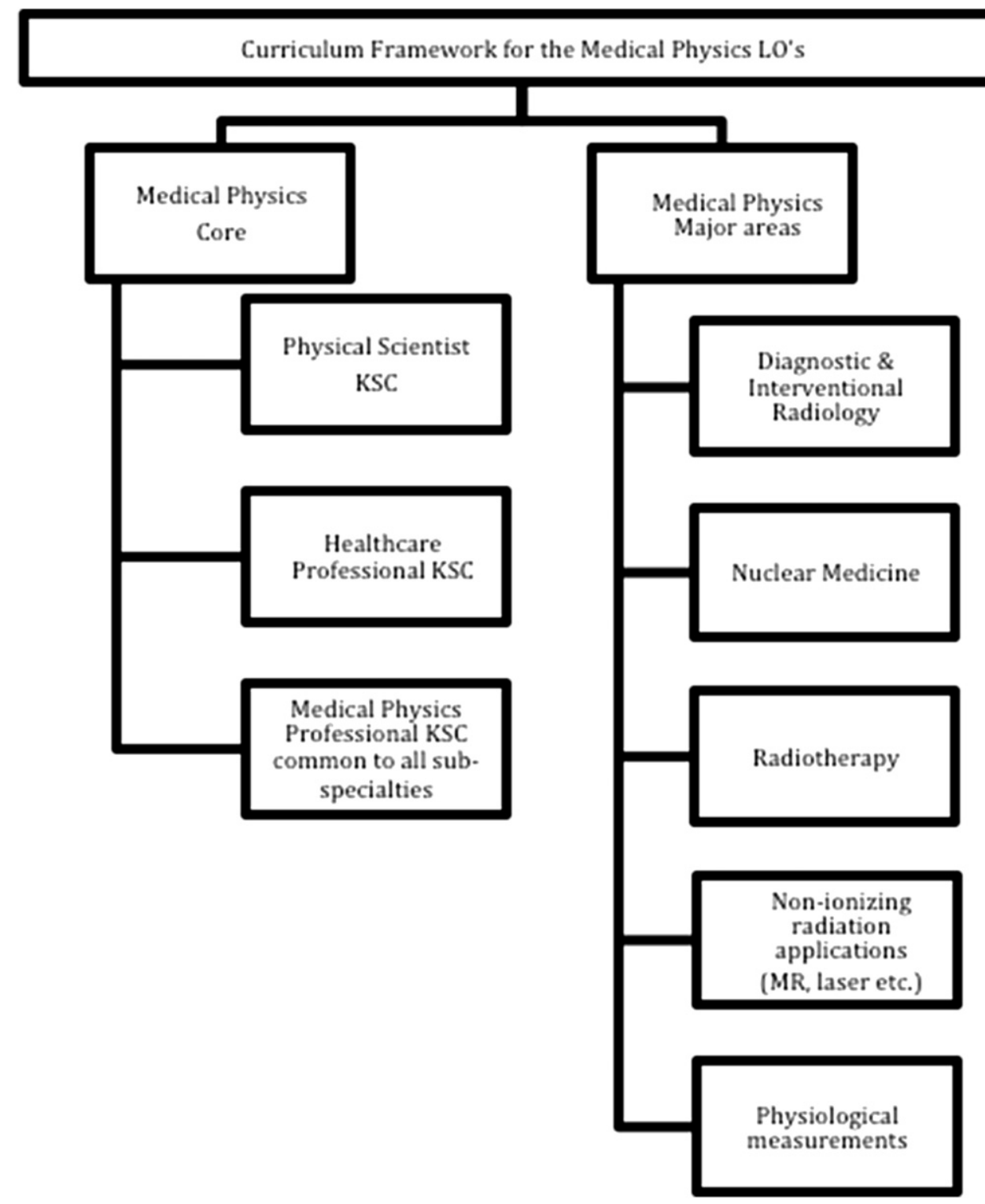

Figure 1 Classification of major areas of the Medical Physics curriculum.

\section{Learning Outcomes for the MP as physical scientist}

\section{Knowledge}

- List the fundamental quantities and dimensions of physics, including use in checking consistency of equations.

- List the common fundamental physical constants.

- List the base and derived SI units.

- List and describe the properties of the common fundamental particles, including mass, charge and spin. Particle-antiparticle annihilation (in depth treatment of positron-electron annihilation).

- List the various forms of energy and types of forces in nature and the properties of their carrier particles.

- Explain the basic principles of quantum theory.

- Describe the structure of the atom and nucleus and define the terms 'isotope' and 'isobar'.
- Explain nuclear and electron energy levels, ionization, nuclear isomers and the Auger effect.

- Describe and explain the structure of the periodic table and chart of the nuclides.

- List and describe the various forms of chemical bonding.

- Explain energy transitions, spectra and the various forms of spectroscopy/spectrometry (including magnetic resonance spectroscopy).

- Describe the band theory of solids with particular emphasis on semiconductors.

- Discuss nuclear stability, list and describe qualitatively the various common modes of radioactive decay (alpha, beta, positron decay, gamma, isomeric transition, electron capture, internal conversion), explain decay schemes, use of decay and secular/transient equilibrium equations. 
- List and describe the main types of nuclear reactions.

- List and describe the basic characteristics of common electronic components and integrated circuits.

- Describe the general design of a measuring instrument.

- List and explain the specifications of measuring instruments including accuracy, SNR, precision, range of measurement, resolution, reliability (repeatability, reproducibility, consistency, stability, ruggedness), sensitivity, specificity, linearity, response time.

- Describe in detail and quantitatively the main types of sensors, their mode of action and response: mechanical (position, velocity, force, pressure, sound and ultrasound), temperature, electric and magnetic fields, voltage, ionizing electromagnetic radiation (including gas-filled, semiconductor, scintillationoptical systems, storage TL phosphor systems, film, diamond, alanine, gel, calorimeter, chemical), nonionizing electromagnetic radiation, ionizing particles, chemical and biochemical.

- Explain quantitatively the following characteristics of ionizing radiation sensors/detectors: pulse height spectrum and energy resolution, counting curves and plateau, detection efficiency and energy response, dead time and temporal resolution.

- Describe and explain in detail equipment used for gamma and X-ray spectrometry.

- Explain the electronic modules used in radiation sensing systems.

- Explain how signals are classified (dimensionality, periodicity, continuity, determinism), acquired, converted to digital form and processed (signals as function of time, spatial coordinates or both, include both continuous and pulse signals).

- Describe and explain the following: temporal/ frequency representation of signals, Fourier transform, statistical description of signals, power spectral density, autocorrelation function, sample (discrete) signals, delta function and its Fourier transform, Fourier transform of a periodic discrete signal (DFT), the FFT, the effect of finite sample intervals, linear processors, impulse response, convolution integral and theorem, various types of filters used in the processing of medical signals.

- Explain the meaning of traceability, calibration and standards.

- List and describe quantitatively and in detail the properties and means of production and control of ionising and non-ionising electromagnetic radiations, particulate radiation beams and ultrasound.

- List and describe the interactions of ionising and non-ionising electromagnetic radiations, particulate charged radiation (electron and ion beams), neutrons and ultrasound and energy absorption/ deposition in inanimate and animate matter (including a quantitative treatment of photon beam attenuation, photoelectric absorption, Rayleigh and Compton scatter, pair-production and the variation of cross-section with photon energy).

- Explain the concepts of cross-section.
- Discuss the characteristics of the common statistical distributions: normal, log-normal, t, Poisson, binomial, uniform.

- List and describe the various forms of uncertainties in the measurement of data and their treatment and the principles of error propagation.

- Explain how quantitative statistical techniques are used to describe and handle data, including the calculation of confidence intervals, combined uncertainties, correlation, regression and hypothesis testing including the influence of sample size.

- Explain the basic principles of modelling and simulation including statistical modelling based on Monte-Carlo techniques.

Skills

- Manage the acquisition, editing, analysis, interpretation, presentation, and reporting of measurement data.

- Communicate clearly results to peers (in the form of notes, resumes, reports, poster, article, oral presentation) at local and international meetings and for research journals.

- Use statistical techniques/tests and software to analyse measurement data and manage associated uncertainties.

- Use modelling and simulation software (e.g. Matlab, SimuLink, IDL, LabView) to solve physics problems including signal and image processing.

- Analyze critically international literature within a given area of research.

- Design and evaluate systems for the rigorous and safe conduct of physical measurements and experiments.

\section{Competence}

- Manage the conduct of experimental work autonomously and in a safe manner.

- Assume responsibility to autonomously: List a set of research objectives worthy of attention, which are realizable given the available resources. Write a literature review article concerning the area of interest. Realize the research objectives by integrating and applying knowledge and skills. Defend results in front of peers.

- Organise networks for research and development within own scientific community.

\section{Learning Outcomes for the MP as a healthcare professional}

\section{Knowledge}

- List and explain the functions of healthcare organizations, describe the way healthcare is organized (internationally, nationally and locally) and discuss principles of clinical governance.

- Utilize accurate medical terminology in communication with other healthcare professionals. 
- Explain relevant sections of the human biological sciences (anatomy, physiology, pathology, cellular and biomolecular science, radiological anatomy) to the level of detail necessary within own area/s of Medical Physics practice.

- Explain and discuss ethical and legal issues in healthcare relevant to the scope of the profession (e.g., research ethics, data protection, privacy, dignity, ethical governance).

- Explain the technological infrastructure required for quality service within own future area/s of Medical Physics.

- Describe and explain the European and national legal frameworks, regulations, guidelines and codesof-practice impacting the profession of MP.

\section{Skills}

- Communicate effectively clinical information, advice, instruction and professional opinion to patients, colleagues, other healthcare professionals, support staff, service users, relatives and carers within own area/s of Medical Physics practice using appropriate terminology.

- Establish the necessary communication links and relations with other healthcare professionals and organizational units related to own area/s of Medical Physics practice.

- Recognize and respond appropriately to own, patients' and relatives' emotional responses.

- Acquire EU Directives, national regulations and guidelines and recommendations from national and international organizations related to Medical Physics.

\section{Competence}

- Practise responsibly within the legal, regulatory and ethical boundaries of the profession.

- Collaborate with other healthcare professionals, support staff and service users, relatives and carers within own area/s of Medical Physics practice.

- Take responsibility for the management of own workload to ensure effective and efficient input to the work of the healthcare team within own area/s of Medical Physics practice.

- Organise the various aspects of the routine service within own area/s of Medical Physics practice.

- Work responsibly within national/local professional codes of practice.

- Take responsibility for appropriate behaviour towards colleagues, patients and relatives as stipulated by organizational policies and national legislation.

- Take responsibility for own input within monodisciplinary and multi-disciplinary research teams.

- Take responsibility for making the best use of available resources to provide optimum healthcare to patients and members of society.

- Assume responsibility for timely action (within own limitations) to prevent and respond to adverse events.
- Assume responsibility to ensure that all activities are based on current best evidence or own scientific research when the available evidence is not sufficient.

- Take responsibility to maintain one's knowledge and skills current through an appropriate continuous professional development programme.

- Facilitate learning of peers, other healthcare professionals, students, and trainees.

- Take responsibility for the development of effective, safe and efficient teams (including multi-disciplinary teams) in own area/s of Medical Physics practice.

\section{Learning Outcomes for the Medical Physicist common to all Medical Physics subspecialties}

The core LO's for the Medical Physicist common to all subspecialties of Medical Physics are subdivided into smaller sub-sections that address the sector of activities for a Medical physicist.

\section{Comprehensive physics problem solving service KSC}

\section{Knowledge}

- Classify the various types of medical devices in terms of function and patient risk.

- Use physics, concepts, principles and theories to describe in detail and quantitatively, the structure, functioning, characteristics and use of the medical devices used in own area/s of Medical Physics.

- Describe in detail and quantitatively the physical properties of the physical agents to be found in the healthcare environment: electrical energy, heat energy, magnetic fields, ionising and non-ionising electromagnetic radiation, ionising particles (electrons and ions), vibration, sound, ultrasound and optical radiation.

- Explain quantitatively the beneficial and/or adverse biological effects of the various physical agents (including ionizing radiation from sealed/unsealed sources) associated with medical devices, the factors influencing the magnitude of the biological effect and the way these can be manipulated to improve clinical outcomes. In the case of ionizing radiation include cell survival curves, linear-quadratic model, absorbed dose, type of radiation (RBE, radiation weighting factor), tissue radiosensitivity (LET, RBE, tissue weighting factor), dose rate, presence of radiosensitisers, oxygen and radioprotectors, age, etc.

Skills

- Use the general concepts, principles, theories and practices of physics to analyze rigorously the research literature concerning the optimal use of medical devices and management of risk from associated physical agents.

- Apply the general concepts, principles, theories and practices of physics to the solution of clinical 
problems concerning the optimal use of medical devices and management of risk from associated physical agents.

- Use physics research skills to develop the evidence base for the optimal use of medical devices and management of risk from associated physical agents when present evidence is insufficient.

- Explain how to use general concepts, principles, theories and practices of physics for the transfer of new medical devices to the clinical environment in an effective, safe and economical manner.

- Use the general concepts, principles, theories and practices of physics to ensure effective and safe practice when using medical devices and the various associated physical agents (including ionizing radiation from sealed/unsealed sources) in own area/s of Medical Physics practice.

\section{Competence}

- Take responsibility for using the general concepts, principles, theories and practices of physics to analyze the research literature concerning the optimal use of medical devices and management of risk from associated physical agents and to transfer relevant published research results to the clinical environment in own area/s of Medical Physics practice.

- Take responsibility for applying the general concepts, principles, theories and practices of physics to the solution of clinical problems concerning the optimal use of medical devices and management of risk from associated physical agents in own area/s of Medical Physics practice.

- Take responsibility to use physics research skills to develop the evidence base for effective and safe clinical actions involving the optimal use of medical devices and management of risk from associated physical agents in own area/s of Medical Physics practice when present evidence is insufficient.

- Take responsibility to use the general concepts, principles, theories and practices of physics for the transfer of new medical devices to own area/s of Medical Physics practice and to facilitate the effective, safe and economical use of the devices.

\section{Physical agents dosimetry service KSC}

\section{Knowledge}

- List and explain the statutory and institutional role of Medical Physics Services with respect to dosimetry of physical agents (including ionising radiation from sealed/unsealed sources).

- Define and explain the physical quantities used to assess beneficial or adverse biological effects for the various types of physical agents (including ionising radiation) and methods for their measurement.

- Define the quantities (including units and interrelationships) used in patient and occupational dosimetry for the various types of ionising and nonionising radiations.

- Describe and explain the structure, operation and advantages/disadvantages of the range of patient dosimeters, personal dosimeters and area monitors available for the various types of ionising and nonionising radiation.

- List and explain the considerations for the choice of dosimeter for patient and occupational exposures for the various types of ionising and non-ionising radiation.

- Explain the principles of dosimetric measurement standards and traceability.

- Explain the fundamental characteristics and limitations of the various models/algorithms used in the quantification of patient and occupational doses from external sources of ionising radiation.

Skills

- Select and operate instrumentation for the measurement of the physical quantities used to assess beneficial or adverse biological effects for the various types of physical agents for the patients, workers and public in own area/s of Medical Physics practice.

- Operate radiation measurement sensors/detectors and interpret the results.

- Develop rigorous dosimetry protocols in own area/s of Medical Physics practice.

\section{Competence}

- Oversee the installation of a laboratory for the measurement of quantities used to assess beneficial or adverse biological effects for the various types of physical agents for the patients, workers and public in own area/s of Medical Physics practice.

- Take responsibility for the selection and operation of instruments for the measurement of the physical quantities used to assess beneficial or adverse biological effects for physical agents in own area/s of Medical Physics practice.

- Take responsibility for the handling, management and maintenance of dosimetry instruments in own area/s of Medical Physics practice.

- Take responsibility for dosimetric investigations and the supervision of dosimetry measurements.

\section{Patient safety/risk management KSC}

\section{Knowledge}

- List and explain statutory and institutional role of Medical Physics Services with respect to patient safety/risk management in the areas of medical devices and physical agents.

- Explain the classification of medical devices based on patient risk. 
- Explain the principles of risk management as applied to medical devices and associated physical agents in the case of patients.

- Explain the possible impact of human factors with regard to the safe use of medical devices and associated physical agents.

- Describe the beneficial and possible adverse biological effects (including mechanisms) to patients of physical agents including the factors impacting the magnitude of the biological effect.

- Explain the difference between deterministic/ stochastic, early/late and teratogenic/genetic effects of the various physical agents in relation to patient risks.

- Explain relevant international, EU, national and local legislation, recommendations and documentation regarding risk from physical agents with the purpose of hazard prevention and emergency preparedness in the healthcare environment with regard to patient safety/ risk management.

- Define the quantities (and units) used in patient radiation protection for both external radiation and internal radionuclides.

- Describe and explain the local procedures for handling adverse incidents related to medical devices and associated physical agents with respect to patient safety.

- Explain how doses to comforters and carers are managed including the use of appropriate dose constraints.

- Explain how research medical exposures are managed including the processes of ethical review and including the use of appropriate dose constraints.

- Describe the process and practical implementation of radiation risk assessments in the context of medical devices and associated physical agents using techniques for the qualitative and quantitative assessment of risk.

- Name and explain the function of the main National, European and International organizations concerned with protection of patients from physical agents (e.g., ICRP, ICNIRP, IAEA, EC).

- List statutory and institutional roles of Medical Physics Services/Experts with respect to patient safety/risk management.

- Define dosimetry and operational quantities (and units) used in patient risk assessment and their use in the radiation protection of patients.

- Describe the requirements for, and the practical implementation of, appropriate systems for the monitoring of dose to patients.

- Describe the principles and practice of contingency planning and the implementation of emergency procedures with respect to patient safety.

- Explain how the application of good radiation safety practice and the use of appropriate techniques and devices are used to optimize patient doses.

- Explain how facilities are designed to ensure protection of patients.
Skills

- Calculate patient risk from measurement data of the dosimetry quantities used to assess adverse biological effects for the various types of physical agents (including ionizing radiation from external/internal sources).

- Apply the various means of dose reduction (appropriate source strengths, exposure time, distance, shielding) and their application in patient dose optimisation.

- Calculate risks to the unborn child in the case of exposure to physical agents (ionizing radiation).

- Perform risk assessment for continued and limited use of equipment that does not meet acceptability criteria.

- Develop an organisational policy to achieve regulatory compliance for patient safety from physical agents in own area/s of Medical Physics practice (including radiation sources and waste, transportation of radioactive materials).

\section{Competence}

- Oversee patient safety/risk management involving medical devices and associated physical agents in own area/s of Medical Physics practice.

- Take responsibility for the optimization of procedures in terms of patient safety in own area/s of Medical Physics practice.

- Take responsibility for the prevention and investigation of adverse incidents.

- Take responsibility for the planning for emergency situations with regard to patient safety in own area/ $s$ of Medical Physics practice.

- Inventorize radiation sources present in the hospital environment with respect to patient safety.

\section{Occupational \& public safety/risk management KSC}

Knowledge

- List and explain statutory and institutional roles of Medical Physics Services/Experts with respect to occupational and public safety/risk management.

- Describe the possible adverse biological effects (including mechanism) to workers/public from physical agents including the factors impacting the magnitude of the biological effect.

- Explain the principles of occupational risk audit and management, hazard prevention and emergency preparedness as applied to physical agents associated with the use of medical devices.

- Explain how the principles of justification, optimisation (including ALARA), and risk limitation are used for occupational and public protection from the deleterious effects of physical agents.

- Explain how facilities are designed to ensure protection of workers and the general public.

- Explain the local procedure for handling adverse incidents related to medical devices and associated physical agents involving workers or public. 
- Explain the scope, objectives, structure and content of formal systems of work ('local rules').

- Define dosimetry and operational quantities (and units) used in occupational/public radiation protection.

- Explain in quantitative terms the various means of dose reduction for external radiation (source strengths, exposure times, distance, shielding) and internal radionuclides with respect to occupational/ public safety.

- State current dose limits for workers and the general public.

- Describe the key considerations for the design of a new facility with regards to occupational/public safety in own area/s of Medical Physics practice.

- Describe the requirements for, and the practical implementation of, appropriate systems for the monitoring of radiation dose to the worker, including extremity doses and dose limits for pregnant and lactating workers, and young workers; and for the public; including selection, management and calibration of devices used to measure such doses, dose records and practical techniques for dose measurement.

\section{Skills}

- Assess safety related human factors and behaviours with regard to occupational and public safety in own area/s of Medical Physics practice.

- Evaluate procedures in terms of occupational/public safety in own area/s of Medical Physics practice.

- Design new facilities in own area/s of Medical Physics practice with due consideration to occupational/public safety including classification of areas.

- Apply good radiation safety practice and the appropriate use of personal protective equipment to minimise internal radiation exposure of workers and the public in own area/s of Medical Physics practice.

- Calculate occupational/public risk from measurement data of the dosimetry quantities used to assess adverse biological effects for the various types of physical agents (including ionizing radiation from external/internal sources).

- Implement appropriate systems for monitoring the radiation dose of the worker (also pregnant and lactating workers), comforters and carers and the public; including selection, management and calibration of devices used to record doses and practical techniques for absorbed dose measurement.

\section{Competence}

- Take responsibility for the management of radiation risk including the implementation of the findings of radiation surveys.

- Manage occupational and public safety/risk management activities in own area/s of Medical Physics practice.

- Manage the optimization of occupational/public risk in own area/s of Medical Physics practice.
- Take responsibility for the management of good and safe practice in the use of sealed/unsealed sources of ionizing radiation in own area/s of Medical Physics practice in relation to occupational and public safety.

- Develop an organisational policy to achieve regulatory compliance for occupational/public safety from physical agents in own area/s of Medical Physics practice (including radiation sources and waste, transportation of radioactive substances, security considerations, storage, shielding, record keeping and audit).

- Take responsibility for the design of a new facility in own area/s of Medical Physics practice taking into consideration occupational/public dose limits, dose constraints and ALARA.

- Inventorize radiation sources present in the hospital environment with respect to occupational/public safety/risk management.

- Take responsibility for regulatory compliance with respect to the management of radiation sources, radiation waste, and the transportation of radioactive substances.

\section{Clinical medical device management KSC}

\section{Knowledge}

- Define/explain terms used in medical device documentation (medical device terminology).

- List the medical devices used in own area/s of Medical Physics practice and explain their purpose, structure and functioning.

- Explain the medical device Directives and associated documentation.

- Explain the meaning of 'acceptability criteria' as applied to medical devices.

- Describe and discuss the principles of medical device design with respect to clinical effectiveness and safety, including human-factors.

- Describe the operational relationships between hospital information systems (HIS) and information systems specific to own area/s of Medical Physics practice (e.g., RIS/PACS for imaging).

- Describe and explain in detail the DICOM standard including its application to own area/s of Medical Physics practice.

- Explain the principles of medical device connectivity and connectivity standards.

- Define and explain the principles of quality, quality assurance, quality control, performance indicators, constancy testing, quality control tests, test frequency, tolerances, and action criteria with respect to medical devices.

- Explain the principles of medical device (including associated software) management including planning, evaluation of clinical needs, preparation of technical specifications for tender purposes, evaluation of tendered devices, procurement, acceptance 
testing, commissioning, constancy testing, maintenance and decommissioning; service contract management.

- Explain and discuss the main properties of biomaterials relevant to medical device design.

- Explain in detail the structure and functioning of the medical devices in own area/s of Medical Physics practice.

Skills

- Use test objects and follow/adapt appropriate National/European/International protocols (e.g., IEC) to measure the performance indicators of medical devices in own area/s of Medical Physics, assess deviations from acceptable levels and the relevance of the deviations for clinical practice and suggest actions for restoring default performance.

- Evaluate technical specifications of commercial devices in own area/s of Medical Physics practice.

- Design and test physical and technical methods for quality control of devices in own area/s of Medical Physics practice.

- Identify sources of device malfunctioning in devices in own area/s of Medical Physics practice.

- Analyze the medical devices used in own area/s of Medical Physics practice and investigate their design, functioning, associated signal/image processing, safety features, typical specifications and performance indicators.

- Autonomously acquire and analyze in detail the literature and user/technical manuals for medical devices in own area/s of Medical Physics practice.

- Carry out acceptance testing, commissioning and constancy testing procedures in own area/s of Medical Physics practice.

- Evaluate and participate in the selection of medical devices in a tender own area/s of Medical Physics practice.

- Utilize RIS, PACS and DICOM in own area/s of Medical Physics practice.

- Develop device specific QC protocols (physical and software test objects and phantoms, data acquisition sequences, data records).

- Adapt national and international QC standards to specific device limitations, where appropriate.

- Apply available systems resources (e.g., RIS, PACS, DICOM data) to QA data elaboration and record.

- Implement cross-institutional quality control procedures for devices.

- Implement cross-calibration procedures between devices.

- Design rooms to accommodate specific devices in own area/s of Medical Physics practice.

\section{Competence}

- Take responsibility for medical device (including software, information systems, PACS) management including planning, evaluation of clinical needs, preparation of technical specifications for tender purposes, evaluation of tendered devices, procurement, acceptance testing, commissioning, quality control (including setting of warning and suspension levels), maintenance, decommissioning and service contract management in own area/s of Medical Physics practice.

- Take responsibility for the maintenance of quality control records.

- Organize infrastructures for distribution, archiving and retrieval of images.

- Organize infrastructures for display and reading of images and for the reporting and archiving of findings.

- Pursue corrective actions with minimum interference with departmental functionality.

- Establish and plan QA/QC procedures in appropriate support of the specific activity of the clinical department.

- Take responsibility for the development of an institutional quality assurance/quality control medical device service and constancy testing teams as required by European and national medical device standard setting bodies in own area/s of Medical Physics practice.

- Take responsibility for the development and ongoing update of quality control protocols for medical devices in own area/s of Medical Physics practice.

- Participate in the installation of new devices in own area/s of Medical Physics practice.

- Negotiate device acceptance with provider and own department management following acceptance tests.

- Organize and manage quality control teams in own area/s of Medical Physics practice.

\section{Clinical involvement KSC}

Knowledge

- Explain the role of healthcare scientists in clinical involvement.

- Describe and explain the principles of anatomy, physiology, biology (including radiobiology) and pathology as related to the main clinical applications in own area/s of Medical Physics practice.

- Describe and explain how medical devices are used for the solution of clinical problems in own area/s of Medical Physics practice.

- Describe and explain the risk/benefit justification of procedures in own area/s of Medical Physics practice.

- Describe and explain the design principles, the relevant legislation issues and approval procedures for clinical trials.

- Describe general indications and contra-indications for the use of devices in own area/s of Medical Physics practice. 


\section{Skills}

- Develop optimized protocols in own area/s of Medical Physics practice.

- Adhere to procedures regarding hygiene.

- Participate in patient preparation and positioning prior to data acquisition.

- Assess patient and operator risks for a given experimental procedure.

- Handle and analyze medical images including the extraction of parametric data/images.

- Set up devices, experiments and protocols for the measurement of physical variables relevant to clinical practice.

\section{Competence}

- Excise the role of the Medical Physicist in clinical involvement within own area/s of Medical Physics practice.

- Advise physician in imaging interpretation and quantification.

- Take responsibility for deriving semi-quantitative and quantitative data for clinical application.

- Take responsibility for optimization of acquisition protocols in both standard and non-standard situations.

- Supervise procedures for paediatric investigations in relation to dose optimisation.

- Advise other healthcare professionals on optimization and safety of individual patient examination/ treatment and examination/treatment protocols.

- Live up to demands imposed by duty of confidentiality, professional secrecy, ethical standards.

- Represent Medical Physics in clinical conferences.

- Share the responsibility for conducting a clinical trial.

- Assume the responsibility of data handling and recording.

- Advise on and take responsibility for the optimization of clinical protocols for medical devices including software.

\section{Development of service quality KSC}

\section{Knowledge}

- Define and explain the principles of quality, continuous quality improvement, quality audit and total quality management as applied to aspects of clinical audits involving medical devices and associated physical agents.

- Explain why the development of service quality for an area of medical practice requires input from various healthcare professionals.

- Describe the intentions and principles of Quality Management systems like ISO 9000.

- Define quality objectives in own area/s of Medical Physics practice.

- Explain the role of the Medical Physicist in the development of service quality in own area/s of Medical Physics practice.

\section{Skills}

- Define, measure and optimize appropriate quality indicators in own area/s of Medical Physics practice.

- Set up a service quality development strategy.

- Measure quality management performance and improvements.

\section{Competence}

- Take responsibility for increasing quality, safety and cost-effectiveness within own area/s of Medical Physics practice whilst being aware that improvement of the service as a whole depends on the inputs of other healthcare professionals.

- Participate in the design and implementation of QA systems in own area/s of Medical Physics practice.

- Assume responsibility for the technical aspects of clinical audits involving medical devices and associated physical agents.

\section{Expert consultancy KSC}

Knowledge

- Explain the role of a consultant.

- Explain the role of scientists as consultants in healthcare.

- Describe the general role of the MP as consultant in own area/s of Medical Physics practice.

- Write reports.

\section{Skills}

- Apply MP consultancy skills to specific scenarios in own area/s of Medical Physics practice.

- Identify relevant conflict-of-interest situations.

- Apply procedures to manage and resolve conflict-ofinterest situations.

- Design educational courses for CPD.

\section{Competence}

- Take responsibility for clinical consultancy services in own area/s of Medical Physics practice commensurate with level of personal expertise.

- Establish a fair conflict-of-interest policy.

- Audit practice and service.

- Audit CPD schemes.

- Consult on practice and service provision.

\section{Education of healthcare professionals and Medical} Physics trainees KSC

\section{Knowledge}

- Discuss the principles of modern education and training of healthcare professionals and trainees (including continuous professional development activities) in the field of Medical Physics, in particular during the introduction of new technologies. 
- Discuss the principles and methods for the establishment of appropriate medical device and physical agents (including radiation) education and training Learning Outcomes for addressing the learning needs of specific healthcare professionals and trainees in specific clinical environments.

- Discuss the application of the principles of knowledge transfer to the case of medical device and physical agents (including radiation) knowledge to specific healthcare professionals and trainees in specific clinical environments.

- Describe the content of appropriate programmes for healthcare professionals and trainees programmes in specific areas of Medical Physics.

\section{Skills}

- Prepare effective and efficient modes of knowledge transfer activities specific to the learning needs of specific healthcare professionals and trainees in specific clinical environments in conjunction with the leaders of the respective healthcare professions.

- Prepare effective modes of assessment appropriate for the various healthcare professions and trainees.

- Carry out own research when the evidence base for education of healthcare professions and trainees is insufficient.

\section{Competence}

- Take responsibility for the education and training (including continuous professional development) of healthcare professionals and trainees within the areas of medical devices and physical agents for specific healthcare professionals in specific clinical environments.

- Take responsibility for the education and training (including continuous professional development) of healthcare professionals and trainees in the optimized use of medical devices/physical agents (including radiation) in own area/s of Medical Physics practice.

- Take responsibility for the education and training of healthcare professionals and trainees in performing QC procedures and for reporting QC data related to medical devices.

- Take responsibility for the education and training of other healthcare professionals and trainees regarding protection from physical agents (including radiation) and including the use of personal ose monitors and personal protection equipment.

\section{Economic issues/Health Technology Assessment (HTA) KSC}

\section{Knowledge}

- Explain the principles of business, strategic planning and cost effectiveness in the case of Medical Physics Services.
- Explain the principles of HTA as applied to medical device technologies and procedures in own area/s of Medical Physics practice.

- List and explain the steps for the carrying out of an HTA, including use of primary data and secondary sources.

- Explain the application of HTA as applied to medical devices and procedures using medical devices.

- List the issues that should be considered in an HTA project in own area/s of Medical Physics practice.

- Explain the value of HTA reports for policy makers at the European, national, regional and facility levels.

- Explain the importance of HTA reports in controlling cost in relation to benefit for the considered technology in own area/s of Medical Physics practice.

- Describe the fundamentals and design models of clinical trials in own area/s of Medical Physics practice.

- Define the roles and responsibilities of all the professionals involved in a HTA project in own area/s of Medical Physics practice.

Skills

- Apply the principles of strategic planning and cost effectiveness in own area/s of Medical Physics practice.

- Perform a systematic review of the existing evidence base to evaluate the clinical effectiveness and safety of a new medical device or new procedure involving medical devices/physical agents.

- Communicate HTA reports to policy makers.

- Design and monitor the Medical Physics components of clinical trial protocols in own area/s of Medical Physics practice.

- Perform statistical analysis and report on clinical trials involving Medical Physics services.

\section{Competence}

- Take responsibility for the development and implementation of a business and strategy plan for Medical Physics Services in own area/s of Medical Physics practice.

- Use the methodologies of HTA to carry out a HTA in conjunction with other healthcare professionals.

- Take responsibility for the technical component of a HTA related to medical devices and/or physical agents.

\section{Innovation}

\section{Knowledge}

- Define innovation as the development of new devices (including software), modification of existing devices (including software) and the development of new techniques using devices for the solution of hitherto unresolved clinical problems. 
- Explain the importance of ongoing horizon scanning for new and emerging technologies.

- Describe the methodology of horizon scanning for new and emerging technologies.

\section{Skills}

- Apply the methodology of horizon scanning (including listing of specific information sources) for new and emerging technologies to own area/s of Medical Physics practice.

\section{Competence}

- Take responsibility for the development of new devices (including software) and modification of existing devices (including software), including their implementation and evaluation in response to clinical needs in own area/s of Medical Physics practice.

\section{Learning Outcomes specific for the Medical Physicist in Nuclear Medicine}

The LO's specific for the Medical Physicist in Nuclear Medicine are subdivided into the same key activities as for the core KSC of the Medical Physicist, i.e., previous Subsection.

\section{Comprehensive physics problem solving service KSC}

\section{Knowledge}

- Describe and explain the beta decay, electron capture, positron decay, positron annihilation, isomeric transitions and their application.

- Describe the principles of radionuclide production with cyclotron, reactors, radionuclide generators and others (including parent isotopes).

- List and describe the radiation detectors used in Nuclear Medicine.

- Illustrate the characteristics of a counting system including the effect of background counts and minimum detectable counts.

- Discuss the characteristics of electronics related to Nuclear Medicine devices.

- Describe the concepts of fundamental detector properties like energy resolution, sensitivity, spatial resolution and temporal resolution and how they affect the performance of Nuclear Medicine devices.

- Explain how statistical techniques are used for radiation measurement in Nuclear Medicine.

- Explain the physical and technological working principles of the imaging devices used in Nuclear Medicine including gamma camera systems, single photon and positron emission tomography systems, combined modality systems and dedicated scanner design.

- List and describe the application of Information and Communication Technology (ICT) to Nuclear Medicine including image storage, image acquisition and processing and file format and secure file transfer.
- Describe the basic concepts of image reconstruction in Nuclear Medicine including analytical and iterative reconstruction techniques.

- Illustrate the basic mathematical concepts used in Nuclear Medicine including linear systems, Fourier analysis and FFT, convolution/deconvolution, curve fitting and function optimization.

- Describe the basic procedures for correction and quantitation and fundamental limits in Nuclear Medicine.

- Explain the concepts of compartmental analysis and its use in Nuclear Medicine.

- Describe up to date Nuclear Medicine literature, scientific reports and national and international recommendations.

Skills

- Identify measurable physical quantities relevant to Nuclear Medicine and realize experiments for their measurement.

- Operate radiation measurement devices/detectors and interpret the results in the context of Nuclear Medicine.

- Design and test physical and technical aids for physical measurements relevant to Nuclear Medicine.

- Realize experiments for the measurement of properties relevant for instrument specific performance assessment, especially with reference to established national and international standards (NEMA, IEC).

- Develop, assess and implement new methods and technologies in Nuclear Medicine.

- Analyze and handle images from a Nuclear Medicine imaging device.

- Extract parametrical information/image from Nuclear Medicine data.

- Calculate biological parameters from Nuclear Medicine images using compartmental modelling.

\section{Competence}

- Equip a laboratory for the measurement of physical quantities relevant to Nuclear Medicine.

- Take responsibility for the good practice in the use of sealed/unsealed sources of ionizing radiation.

- Take responsibility for the inventory of sealed radiation sources present in the laboratory and in the hospital environment.

- Support the measurement of physical quantities relevant to Nuclear Medicine.

- Take responsibility for the handling, management and maintenance of radiation measurement devices.

- Supervise image reconstruction and image handling procedures.

Internal radionuclide dosimetry service KSC

\section{Knowledge}

- Describe how dosimetric calculations may be made in diagnostic and therapeutic practice, and how this conditions the level of accuracy required. 
- Explain the MIRD scheme, understanding its development and the fundamental characteristics and limitations of the formalism, and how this governs its usage.

- Explain the role of the ICRP in the development of the dosimetric formalism, including use of the ICRP reference phantoms.

- List the various equipments and devices required within the context of patient dosimetry including probes, well counters, activity calibrators, gamma cameras \& PET scanners (including hybrid systems).

- Define the reproducibility of the patient positioning and list the methods assuring reproducibility in image quality.

- Describe calibration factors including phantom set up and measurements for dosimetry specific image quantification.

- Describe the influence of the equipment settings (e.g. choice of energy windows, collimators, scan duration, count statistics) on dosimetry results and how temporal sampling (scheduling of image acquisition) affects the results obtained.

- Describe and explain the role and influence of attenuation, background and scatter corrections/ geometry/shielding/collimators/dead time correction, partial volume effect, cross-talk, when relevant, in all devices involved in activity determination for dosimetry.

- Describe the influence of the reconstruction method and the respective parameters used in processing parameters in PET/SPECT (e.g. cut-off frequency, number of iterations, number of subsets, post-filtering type and parameters) on dosimetry results.

- Explain how cumulated activity is derived from time-activity curve data by appropriate methods, including curve fitting algorithms and compartmental analysis.

- Explain how standard geometric models may be made patient-specific by scaling to individual body mass, organ volume/mass and tissue density.

- List the main types of computer codes used for dose calculation.

- List methods for determining patient-specific organ masses including the respective errors and explain the difference between morphological and functional volume of organs or lesions.

- Describe the principles of tumour dosimetry.

- Explain the fundamental limitations of dosimetry at the organ level, for instance in deriving tumour dosimetry, taking into account activity and density heterogeneities.

- Describe the application and use of techniques for absorbed dose determination at the sub-organ, voxel and cellular level, in the context of molecular radiotherapy.

- Describe how Dose-Volume-Histograms or isodose curves are calculated and what results should be provided.
Skills

- Distinguish between the requirements for radiation protection dosimetry and the need for patientspecific dosimetry in a therapeutic setting.

- Develop skills for designing optimised dosimetry protocols and calculation procedures for molecular radiotherapy.

- Develop skills for assessing the requirements for quantitative imaging and/or other measurements for dosimetric purposes.

- Develop skills for calculating cumulated activity (incl. curve-fitting techniques and use of compartmental modelling).

- Develop methods for assuring reproducibility of dosimetry assessments.

- Perform dosimetric calculations using the MIRD formalism, including the appropriate adaptation of standard models and data to achieve patient-specific estimates.

- Delineate the differences between the methods used for calculating $\mathrm{S}$ factors (point-kernel vs. Monte-Carlo).

- Delineate how accurately organ masses are determined using different modalities.

- Calculate whole body, organ and tissue absorbed doses (or effective doses when relevant) from clinical data, e.g. using tools such as OLINDA.

- Develop skills for applying the correct radiobiological concepts.

- Determine when voxel-based dosimetry and use of dose-volume histograms is appropriate.

\section{Competence}

- Take responsibility for dosimetric investigations including the supervision of dosimetric measurements.

- Take responsibility and supervise the development of appropriate dosimetry protocols including quantitative imaging aspects, time-sampling, time-activity curves derivation and absorbed dose calculations.

- Advise on the statutory and institutional requirements for Medical Physics Services in Nuclear medicine with respect to patient safety/risk management in a therapeutic context.

Patient dose optimization KSC

Knowledge

- Explain the concepts of absorbed dose and effective dose and the ALARA principle as applied to patient dose optimisation in Nuclear Medicine.

- Describe how diagnostic and therapeutic medical exposures are managed in the context of Nuclear Medicine, including the application of Diagnostic Reference Levels and optimisation of dose through prescription of the activity, the procedure and protocol to be used. 
- Explain how standard exposures and procedures can be modified in special cases, e.g. the pregnant patient, the lactating patient, and paediatric patients.

- Explain how research medical exposures are managed in the context of Nuclear Medicine, including the processes of ethical review and clinical trials administration and governance and the use of appropriate dose constraints.

- Describe the process and practical implementation of radiation risk assessments in the context of Nuclear Medicine using techniques for the qualitative and quantitative assessment of risk, and the assessment of absorbed dose to the patient arising from both internal and external sources of exposure.

- Explain radiation quantities related to CT devices as part of hybrid systems.

\section{Skills}

- Estimate relevant activity to inject to paediatric patients according to international recommendations.

- Systematize the inclusion of dosimetry reports based on injected activity and ICRP data for diagnostic procedures in patient medical records.

- Estimate foetus effective dose in pregnant patients.

- Apply relevant guidance document in dosimetry reporting for molecular radiotherapy.

- Interpret radiation quantities related to CT devices as part of hybrid systems and apply these appropriately to dose optimization.

\section{Competence}

- Take responsibility for patient dose optimisation within the Nuclear Medicine facility.

- Take responsibility for ensuring the optimal patient radiation protection applying the ALARA principle.

- Advise on the statutory and institutional requirements for Medical Physics Services in Nuclear medicine with respect to patient safety/risk management for diagnostic \& therapeutic procedures.

\section{Occupational/public dose optimisation KSC}

\section{Knowledge}

- Describe the key considerations when designing a new Nuclear Medicine facility that optimise radiation safety of workers and the public (including classification of radiation areas); to include diagnostic Nuclear Medicine imaging with PET and multimodality imaging, non-imaging or in-vitro laboratory procedures, radionuclide therapy, and radiopharmaceutical production including cyclotron PET tracer production.

- Explain the need for, and use of radiation risk assessments in Nuclear Medicine using qualitative and quantitative risk assessment, and the assessment of absorbed dose to workers and public arising from internal and external exposure.

- Describe the requirements for regulatory compliance with respect to the management and use of sealed and unsealed radiation sources, including security considerations, requirements for storage, shielding, record-keeping and audit.

- Describe the requirements for regulatory compliance with regard to the management and disposal of radioactive waste and the transportation of radioactive substances.

- Explain the nature and sources of internal and external radiation exposure and the relevant dose limits in Nuclear Medicine for the worker, including extremity doses and dose limits for pregnant and lactating workers, and young workers, and the public, and dose constraints for comforters and carers.

- Explain how therapeutic exposures are managed in both inpatient and outpatient contexts.

- Describe factors for optimizing acquisition/processing procedures to decrease $\mathrm{CT}$ dose in combined modalities.

- Describe and explain the ALARA principle.

- Describe appropriate systems for monitoring absorbed dose to pregnant and lactating workers, young workers, and the public, including selection, management and calibration of devices used to record absorbed doses and practical techniques for absorbed dose measurement.

- Explain the practical application of the principles of time, distance and shielding to the radiation safety of the worker and public from Nuclear Medicine practices.

- Explain how good radiation safety practice and appropriate personal protective equipment minimises internal radiation exposure of the worker and public in Nuclear Medicine.

- Describe the role of designated radiation protection officers in the management of systems for radiation safety in Nuclear Medicine.

- Explain the purpose and implementation of formal systems of work ('local rules') with regard to radiation safety in Nuclear Medicine.

- Explain the nature of contamination and practical measures required to affect environmental and personal decontamination in Nuclear Medicine; its relevance to radiation safety of the worker and public, and the principles, systems and precautions required to minimise the hazard.

- Describe the principles of contingency planning and emergency procedures in Nuclear Medicine.

- Describe suitable processes for the reporting of radiation incidents in Nuclear Medicine, using root cause analysis and other tools to determine the underlying cause(s).

\section{Skills}

- Design a new Nuclear Medicine facility; including diagnostic Nuclear Medicine imaging with PET and multi- 
modality imaging, non-imaging or in-vitro laboratory procedures, radionuclide therapy, and radiopharmaceutical production including cyclotron PET tracer production.

- Classify appropriately radiation areas within a Nuclear Medicine facility.

- Develop a detailed organisational (hospital) policy to support the radiation safety of staff and public in Nuclear Medicine.

- Develop an organisational (hospital) policy for regulatory compliance with regard to the management of radiation sources and radiation waste.

- Develop a policy for regulatory compliance with regard to the transportation of radioactive substances.

- Implement appropriate systems for monitoring the absorbed dose of the worker (including pregnant and lactating workers), comforters and carers and the public; including selection, management and calibration of devices used to record absorbed doses and practical techniques for absorbed dose measurement.

- Apply the concept of ALARA and the principles of time, distance and shielding to the radiation safety of the worker and public in Nuclear Medicine.

- Apply good radiation safety practice and the appropriate use of personal protective equipment to minimise internal and external radiation exposure of workers and the public arising from Nuclear Medicine.

- Develop formal systems of work ('local rules') with regard to radiation safety in Nuclear Medicine.

- Investigate radiation incidents in Nuclear Medicine to determine the cause(s) and recommending appropriate remedial action(s).

- Develop contingency plans for emergency procedures relevant to Nuclear Medicine.

\section{Competence}

- Take responsibility for the design of a Nuclear Medicine facility; including diagnostic Nuclear Medicine imaging with PET and multi-modality imaging, non-imaging or in-vitro laboratory procedures, radionuclide therapy, and radiopharmaceutical production (aspects related to radiation) including cyclotron PET tracer production.

- Take responsibility for the classification of radiation areas within a Nuclear Medicine facility.

- Take responsibility for the implementation of a detailed organisational (hospital) policy to support the radiation safety of staff and public in Nuclear Medicine.

- Take responsibility for regulatory compliance with respect to the management of radiation sources and radiation waste.

- Take responsibility for regulatory compliance with regard to the transportation of radioactive substances.

- Take responsibility for the implementation of formal systems of work ('local rules') with regard to radiation safety in Nuclear Medicine.
- Take responsibility for the radiation safety of staff and public, including systems for monitoring the dose of the worker and public, and practical measures to support this - ALARA, the principles of Time Distance Shielding, good radiation safety practice and the use of personal protective equipment.

- Take responsibility for the management of radiation risk including the implementation of the risk assessment findings.

- Take responsibility for the implementation of contingency plans for emergency procedures relevant to Nuclear Medicine.

- Investigate radiation incidents in Nuclear Medicine to determine the cause(s) and recommending appropriate remedial action(s).

\section{Clinical Nuclear Medicine device management KSC}

\section{Knowledge}

- List statutory and institutional requirements for Medical Physics Services in Nuclear Medicine with respect to medical device management.

- List the fundamental components of medical devices used in Nuclear Medicine.

- Define the specifications of a Nuclear Medicine imaging device for tender purposes, generally and as tailored to clinical requirements for the device.

- Specify acceptability criteria for medical devices used in Nuclear Medicine both generally and with respect to their specific clinical usage.

- List the combined modality imaging systems and illustrate possible applications in Nuclear Medicine.

Skills

- Design rooms and infrastructures of a department of Nuclear Medicine.

- Evaluate Nuclear Medicine devices in a tender both generally and as required with respect to clinical requirements for the device.

- Design and test physical and technical aids for examination/treatment of patients.

- Adapt quality control protocols to the specific type/ model of medical devices used in Nuclear Medicine.

- Analyze the results of quality control protocols and assess the performance of a device and compare the results with reference values as indicated by the manufacturer and/or local, National, European and other authorities/bodies.

- Design and test physical and technical methods for the assessment of equipment used in Nuclear Medicine procedures.

\section{Competence}

- Take responsibility for the statutory and institutional requirements for Medical Physics Services in Nuclear Medicine with respect to medical device management. 
- Autonomously organize the quality assurance service as required by local, national, European and other authorities/bodies.

- Support Nuclear Medicine staff with physicaltechnical guidelines.

- Organize infrastructures for display and reading of the examination and for the reporting and archiving of findings.

- Organize infrastructures for distribution, archiving and retrieval of Nuclear Medicine images.

- Supervise purchase, acceptance, commissioning/decommissioning, maintenance and quality control of the equipment and systems in a Nuclear Medicine department.

- Supervise installation of new equipment.

- Decide if actions are required on a medical device to restore default performance.

\section{Clinical involvement KSC}

\section{Knowledge}

- Describe the principles of anatomy, physiology, biology, radiobiology and pathology as related to the main clinical application of Nuclear Medicine diagnostic and therapeutic techniques.

- Describe the general role of Nuclear Medicine procedures in diagnosis, therapy and treatment response evaluation.

- Explain how the Nuclear Medicine devices are used for the solution of a clinical problem.

- Describe the principle of radiopharmaceutical preparation and associated quality control.

- Describe the principles of radiopharmaceutical biodistribution in normal organ and target tissues.

- Describe the fundamentals of molecular radiotherapy.

- Explain the fundamentals of the use of Nuclear Medicine procedures in EBRT planning.

- Describe general indications and contra-indications for Nuclear Medicine procedures.

- Describe diagnostic procedure and clinical procedure guidelines.

- Describe protocol optimization principles.

- Describe the risk/benefit justification of Nuclear Medicine diagnostic and therapeutic procedures as related to the radiation exposure risk.

- Explain the interactions/synergism between chemotherapy, EBRT and molecular radiotherapy.

- Illustrate methodologies for the measurement of the lesions response to therapy.

- List laboratory and imaging procedures to evaluate organ toxicity.

- Illustrate dose limiting toxicity classification and quantification.

\section{Skills}

- Develop optimized imaging and therapeutic
protocols.
- Design a treatment plan.

- Show how Nuclear Medicine data are used in radiotherapy planning.

- Analyze how molecular radiotherapy could impact on other treatment modalities.

- Analyze critically protocol proposal (i.e. feasibility, safety...).

- Analyze the limits of acceptability of clinical Nuclear Medicine procedures.

- Calculate patients and operators doses and consequent risks for a given clinical or experimental procedure.

\section{Competence}

- Advise Nuclear Medicine physicians in imaging interpretation and quantification.

- Take responsibility for deriving semi-quantitative and quantitative data for clinical application.

- Advise on different treatment schedule options.

- Advise on the most appropriate Nuclear Medicine procedure with respect to risk/benefit ratio.

- Take responsibility for optimization of acquisition protocols in both standard and non-standard situations.

- Supervise procedures for paediatric investigations.

- Assume the responsibility of data handling and recording.

- Advise on and take responsibility for the optimization of clinical protocols for Nuclear Medicine quipment including software.

\section{Development of service quality KSC}

\section{Knowledge}

- List the physical and chemical properties of radionuclide compounds selected to implement quality control (QC) and their radioprotection implications.

- Describe the institutional framework of quality assurance (QA) activity and regulation.

- Describe duties and responsibility of other health professionals involved in QA activities.

- Explain the principles of quality control for production of isotopes and synthesis of radiopharmaceuticals.

- Describe the principles of QC for Nuclear Medicine devices, such as gamma probes, well counters, dose calibrators, gamma cameras, SPECT, PET, hybrid systems etc.

- Describe device QC for dosimetry specific image quantification.

- Describe QC measures in sequential imaging (several patient visits).

- Describe QC for synergistic use of data from various modalities.

Skills

- Interpret and apply local radioprotection rules as applicable to QC procedures. 
- Acquire and analyze equipment instruction manuals

- Develop equipment specific QC procedures (physical and software phantoms, data acquisition sequences, data record).

- Adapt national and international QC standards to specific equipment limitations, where appropriate.

- Assess accuracy and reproducibility of radionuclide solutions preparation.

- Assess deviations of performance parameters from reference levels and interpret their relative relevance.

- Setup a feedback system for improving quality after non-conformities, deviations and accidents.

- Measure quality management performance and improvements.

- Implement cross-institutional quality control procedures.

- Implement cross-calibration procedures between devices.

- Understand the concept of reference sources, both internal and external for absolute radioactivity determination (traceability, reference laboratories, accuracy, etc.).

- Perform a documented risk assessment for equipment not meeting acceptability criteria.

\section{Competence}

- Take responsibility for design and implementation of a QC/QA framework in a Nuclear Medicine department.

- Establish QA procedures in appropriate support of the specific activity of the Nuclear Medicine department.

- Negotiate equipment acceptance with provider and NM department after acceptance testing.

- Plan and perform QC procedures in accordance with the Nuclear Medicine department activity.

- Pursue corrective actions with minimum interference with the Nuclear Medicine department functionality.

- Train and supervise technologists in performing QC and in their reporting.

- Organize and supervise the preparation of radioactive sources for QC procedures.

- Define warning and suspension levels for equipment.

- Advise on and take responsibility for quality assurance (including criteria for corrective action) of Nuclear Medicine equipment (including software and Information Systems/PACS).

- Supervise the maintenance and service of equipment.

\section{Expert consultancy KSC}

Knowledge

- Describe the general role of the MP as consultant in Nuclear Medicine.

\section{Skills}

- Apply MP consultancy skills to specific scenarios in Nuclear Medicine.

\section{Competence}

- Take responsibility for clinical consultancy services in Nuclear Medicine commensurate with level of personal expertise.

\section{Education in NM of healthcare professionals and trainees} KSC

Knowledge

- Explain the radiation protection principles underpinning current referral criteria for Nuclear Medicine procedures.

- Describe appropriate programmes for staff training in radiation safety in Nuclear Medicine.

- Explain the underpinning physics of Nuclear Medicine essential for other members of staff comprising the multi-disciplinary Nuclear Medicine team.

- Explain the working principles of imaging devices used in Nuclear Medicine to other members of staff comprising the multi-disciplinary Nuclear Medicine team.

- Explain the optimized use of medical devices used in Nuclear Medicine, and the use of new equipment and/or methods.

Skills

- Develop appropriate programmes for staff training in radiation safety with regard to Nuclear Medicine.

- Develop appropriate programmes for staff training in the underpinning physics of Nuclear Medicine.

- Develop appropriate programmes for staff training in the working principles of imaging devices used in Nuclear Medicine.

- Develop appropriate programmes for staff training in the optimized use of medical devices used in Nuclear Medicine, and the use of new equipment and/or methods.

\section{Competence}

- In conjunction with other healthcare professionals in Nuclear Medicine, take responsibility for ensuring that referrers are knowledgeable of current referral criteria for Nuclear Medicine procedures.

- Train staff to implement patient dose optimisation within the Nuclear Medicine facility.

- Take responsibility for the delivery of appropriate programmes for staff training in radiation safety with regard to Nuclear Medicine.

- Teach healthcare professionals and trainees the physical principles of radionuclide decay, production and handling.

- Teach healthcare professionals and trainees the working principles of imaging devices used in Nuclear Medicine.

- Train healthcare professionals and trainees in the optimized use of medical devices used in Nuclear Medicine.

- Supervise and train healthcare professionals and trainees in the use of new equipment and/or methods. 


\section{Economic Issues/HTA KSC}

Innovation KSC

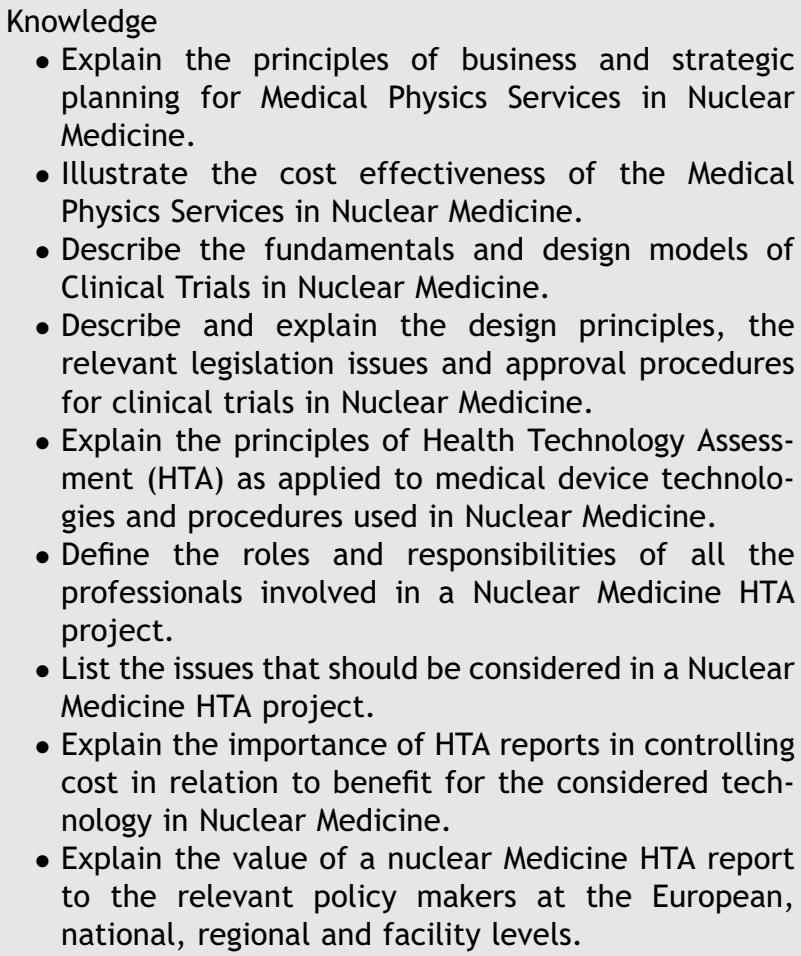

- Explain the principles of business and strategic planning for Medical Physics Services in Nuclear Medicine.

- Illustrate the cost effectiveness of the Medical Physics Services in Nuclear Medicine.

- Describe the fundamentals and design models of Clinical Trials in Nuclear Medicine.

- Describe and explain the design principles, the relevant legislation issues and approval procedures for clinical trials in Nuclear Medicine.

- Explain the principles of Health Technology Assessment (HTA) as applied to medical device technologies and procedures used in Nuclear Medicine.

- Define the roles and responsibilities of all the professionals involved in a Nuclear Medicine HTA project.

- List the issues that should be considered in a Nuclear Medicine HTA project.

- Explain the importance of HTA reports in controlling cost in relation to benefit for the considered technology in Nuclear Medicine.

- Explain the value of a nuclear Medicine HTA report to the relevant policy makers at the European, national, regional and facility levels.

\section{Skills}

- Develop a business and strategy plan for Medical Physics Services in Nuclear Medicine.

- Interpret the statutory and institutional requirements of the Medical Physics Services in Nuclear Medicine.

- Design and Monitor the Medical Physics components of clinical trial protocols in Nuclear Medicine.

- Perform statistical analysis and report on Clinical Trials involving Medical Physics services in Nuclear Medicine.

- Assemble a suitable team for a specific HTA project in Nuclear Medicine.

- Conduct the technical components of an HTA project in Nuclear Medicine.

\section{Competence}

- Take responsibility for the development and implementation of a business and strategy plan for the Medical Physics Services in Nuclear Medicine.

- Consult on the design of clinical trials involving medical devices in Nuclear Medicine.

- Take responsibility for the evaluation of a clinical trial protocol.

- Share the responsibility for conducting a clinical trial.

- Advise on relevant aspects of the ethical review of a clinical trial.

- Take responsibility for the technical components of an HTA project in Nuclear Medicine.

\section{Knowledge}

- Describe the methodology of horizon scanning for new and emerging technologies in Nuclear Medicine.

Skills

- Integrate new devices (including software) in an existing infrastructure.

- Apply the methodology of horizon scanning (including listing of specific information sources) for new and emerging technologies in Nuclear Medicine.

\section{Competence}

- Take responsibility for the development of new devices (including software) or modification of existing devices (including software) in response to clinical needs in Nuclear Medicine.

- Take responsibility for the definition of the experimental set-up and the development of new phantoms for new devices performance assessment.

\section{Conclusions}

This curriculum is intended to be a guideline for the harmonization of Medical Physicists education and training in Nuclear Medicine throughout Europe. Several organizations and scientific associations documents have been instrumental for preparing this curriculum, In particular the IAEA documents available at the IAEA website (http;// Www.iaea.org) have been particularly valuable [17-19]. This curriculum has been given in terms of LO's; in order to be fully implemented in practice, it has to be converted in a syllabus that comprises both the theoretical arguments and the practical training.

In the appendices $A$ and $B$ two examples are given on how the LO's described in Section 4.4 could be related to a more conventional syllabus in Nuclear Medicine, The key activities that have been chosen are Comprehensive physics problem solving service and Clinical Involvement and are reported in appendix $A$ and $B$, respectively, both in terms of the theoretical part and the practical training.

\section{Acknowledgements}

The support of the EU under the funded project ("Guidelines on Medical Physics Expert Project", TREN/09/NUCL/ SI2.549828) and the collaboration of the members of the above Consortium is gratefully acknowledged.

\section{Appendix A \\ Curriculum Items: example 1 - comprehensive physics problem solving service for Medical Physicists working in Nuclear Medicine}




\section{Theoretical part}

Medical devices

Methods of acquiring physiological and anatomical measurements

Blood pressure, ECG, EMG, EEG, NIR, laser, US, strain gauge, ionising radiation etc.

Basal function of medical devices, electrical safety

Active vs. passive devices (pumps, stimulators, pacemakers, prosthesis, etc.)

Physiological measurements

Review of Radiation Physics

Atomic \& nuclear Physics

Radioactivity and radioactive decay

Poisson distribution

Production of radioactive isotopes

Radiation Physics, interaction of photons and charged particles with matter

Detection of ionising radiation

Gas filled detectors, semiconductor based devices, scintillation detectors

Measurement of events from ionizing radiation interactions (energy, time, position, etc.)

\section{Radiation measurement and instrumentation}

Imaging and non-imaging measurement devices (monitors, survey meters, etc.): principles, electronics, data acquisition and calibration

Semiconductor based devices: principles, electronics, data acquisition and calibration

Performance measurement of instrumentation for Nuclear Medicine:

Sensitivity

Energy, Spatial and Temporal resolution

NM instrumentation (including gamma camera, PET, SPECT, hybrid systems)

Principles, instrumentation

Acceptance testing

Quality control

Biological effects of physical agents (without ionising radiation)

Basal physiology

Human homeostasis

Regulation of fluid- and electrolyte-balance and temperature

Common physical agents: mode of action

Dosimetry

Basic quantities (absorbed dose, kerma, exposure, etc.)

Methods for patient dosimetry (models, calculation and measurement)

Dosimetry standards

Instrumentation for dosimetry: acceptance testing and QC

Data acquisition, processing and integration

Linear systems, Fourier transforms, convolution, deconvolution, curve fitting and optimisation

Reconstruction algorithms
Correction methods (scatter, attenuation, resolution)

Filtering

Partial volume effect

Segmentation, co-registration and image fusion

Compartmental and pharmacokinetic modelling.

Information technology

Basic concepts of computers and networks

IT standards for information exchange in Healthcare (DICOM, HL7)

Software for image processing, analysis and visualisation

\section{Practical training}

A basic practical training could be set up as specified in the IAEA Training Course Series No. 50, "Clinical Training of Medical Physicists specialising in Nuclear Medicine" [19]. This course is considered to be a minimum prerequisite for the practical training. Some of the knowledge, skills and competencies listed in this document go beyond the suggestions for training of the IAEA. Therefore, an advanced practical training curriculum should be compiled that surpasses the contents of the basic IAEA training course. In this curriculum local competencies and expertise should also be included.

\section{Appendix B}

Curriculum items: example 2 - clinical involvement for Medical Physicists working in Nuclear Medicine

\section{Theoretical part}

Fundamentals of Human Anatomy, Physiology, Pathology and Radiobiology

Medical terminology

Fundamentals of anatomy and physiology

Orientation, planes and directions

Anatomy at the biochemical, cellular and tissue level Nervous system

Blood and the cardiovascular system

Lymphatic system and Immunity

Respiratory system

Hepatobiliary system

Digestive system

Kidneys and urinary tract

Reproductive system and embryological development Endocrine system

Immune system

Musculo-skeletal system and the skin

Further fundamentals of physiology

Metabolic function

Sensory function

Neurophysiology and cerebral function

Regulation of fluids, electrolytes and temperature

Fundamentals of pathology

Normal tissue and cell function

Infectious processes and disorders of the immune system

Oncogenesis 
Fundamentals of radiobiology

Cell cycle

Genes and mutations

Biochemical damage and radiosensitivity; radioprotectors

Cell survival curves

Dose rate response

Acute radiation sickness

Chronic radiation effect

Radiation-induced cancers

Radiation-embryology

Justification

Nuclear Medicine procedures

Principles of the radioactive tracer method in Nuclear Medicine

Radiopharmaceuticals for the assessment of normal and abnormal function

Quantitative parameters for physiological function; uptake, retention, flow, clearance etc.

Compartmental modelling

Data extraction for the determination of functional parameters

Principles and techniques for the evaluation of treatment response

Assessment of normal organ toxicity and determination of dose limiting toxicity

The role of functional imaging in radiotherapy planning

Clinical procedures for Diagnostic Investigations

Clinical procedures, indications and contra-indications Use of imaging and non-imaging instrumentation for clinical procedures

Clinical protocols; principles for optimisation and procedure guidelines

Paediatric procedures; optimisation and techniques

Prevention and recognition of artefacts

Interpretation of image data and quantitative findings

Risk/benefit analysis and the process of justification

Radiopharmaceuticals for Nuclear Medicine

Radionuclide production techniques

Use of radionuclide generators

Principles and practice of GMP production

Radiopharmaceutical production techniques and quality control requirements

Artefacts and adverse reactions

Molecular radiotherapy

Radiopharmaceuticals and clinical protocols, optimisation and planning

Use in combination of chemotherapy, EB radiotherapy and molecular radiotherapy

Prerequisites for dosimetry for molecular radiotherapy

\section{Practical training}

A basic practical training could be set up as specified in the IAEA Training Course Series No. 50, "Clinical Training of Medical Physicists specialising in Nuclear Medicine" [19]. This course is considered to be a minimum prerequisite for the practical training. Some of the knowledge, skills and competencies listed in this document go beyond the suggestions for training of the IAEA. Therefore, an advanced practical training curriculum should be compiled that surpasses the contents of the basic IAEA training course. In this curriculum local competencies and expertise should also be included.

\section{Appendix C}

Abbreviations and acronyms

\section{AAPM American Association of Physicists in} Medicine

ALARA As Low As Reasonably Achievable

BSS Basic Safety Standards

CPD Continuing Professional Development

CT Computed Tomography

DFT Discrete Fourier Transform

DICOM Digital Imaging and Communications in Medicine

DRL Diagnostic Reference Level

EANM European Association of Nuclear Medicine

EBRT External Beam Radiotherapy

EC European Commission

ECG Electro-Cardio-Graphy

ECTS European Credit Transfer System

EEG Electro-Encephalo-Graphy

EFOMP European Federation of Organisations for Medical Physics

EJNMMI European Journal of Nuclear Medicine and Molecular Imaging

EMG Electro-Magneto-Graphy

ESTRO European Society of Radiotherapy \& Oncology

EU European Union

EQF European Qualifications Framework

FFT Fast Fourier Transform

HIS Hospital Information System

HL7 Health Level 7

HTA Health Technology Assessment

IAEA International Atomic Energy Agency

ICRP International Commission on Radiation Protection

ICNIRP International Commission on Non-Ionising Radiation Protection

ICT Information and Communication Technology

IDL Interface Description Language

IEC International Electrotechnical Commission

ILO International Labour Organisation

IOMP International Organisation of Medical Physics

ISCO International Standard Classification of Occupations

ISO International Standards Organisation

KERMA Kinetic Energy Released per MAss unit

KSC Knowledge, Skills, Competence

LET Linear Energy Transfer

LO Learning Outcomes

MED Medical Exposures Directive

MIRD Medical Internal Radiation Dose 


$\begin{array}{ll}\text { MP } & \text { Medical Physicist } \\ \text { MPE } & \text { Medical Physics Expert } \\ \text { NEMA } & \text { National Electrical Manufacturers Association } \\ \text { NIR } & \text { Non lonizing Radiation } \\ \text { PACS } & \text { Picture Archiving and Communication System } \\ \text { PET } & \text { Positron Emission Tomography } \\ \text { QA } & \text { quality assurance } \\ \text { QC } & \text { quality control } \\ \text { RBE } & \text { Relative Biological Effectiveness } \\ \text { RIS } & \text { Radiology Information System } \\ \text { SI } & \text { Système Internationale } \\ \text { SNM } & \text { Society of Nuclear Medicine } \\ \text { SNR } & \text { Signal to Noise Ratio } \\ \text { SPECT } & \text { Single Photon Emission Tomography } \\ \text { TL } & \text { Thermoluminescence } \\ \text { US } & \text { Ultrasound }\end{array}$

\section{References}

[1] Council directive $96 / 29$ /euratom of 13 May 1996, laying down basic safety standards for the protection of the health of workers and the general public against the dangers arising from ionising radiation. Official Journal of the European Communities; 29.06.1996. No. L-159, p. 1.

[2] Directive $97 / 43$ /euratom of 30 June 1997 on health protection of individuals against the dangers of ionising radiation in relation to medical exposure, vol. 22. Official Journal of the European Communities; 9 July 1997. No. L 190 p. 5.

[3] EFOMP policy statement no. 9: radiation protection of the patient in Europe: the training of the medical physics expert in radiation physics or radiation technology. Physica Medica 1999;XV(3):149-53.

[4] EFOMP policy statement no. 8: continuing professional development for the medical physicist. Physica Medica 1998;XIV(2):81-3.

[5] EFOMP policy statement no. 6: recommended guidelines of national registration schemes for medical physicists. Physica Medica 1995;XI(4):157-9.
[6] EFOMP policy statement no. 10: recommended guidelines on national schemes for continuing professional development of medical physicists. Physica Medica 2001;XVII(2):97-101.

[7] EFOMP policy statement no. 12: the present status of medical physics education and training in Europe. New perspectives and EFOMP recommendations. Physica Medica 2010;26:1-5.

[8] Eudaldo T, Huizenga H, Lamm IL, McKenzie A, Milano F, Schlegel W, et al. Guidelines for education and training of medical physicists in radiotherapy. Recommendations from an ESTRO/EFOMP working group. Radiotherapy and Oncology 2004;70:125-35.

[9] European Commission. Radiation protection 116. Guidelines on education and training in radiation protection for medical exposures. Luxembourg: Directorate General Environment, Nuclear Safety and Civil Protection; 2000.

[10] Paganetti Harald. Private communication. IOMP; 2010.

[11] EFOMP policy statement 7: criteria for the staffing levels in a medical physics department. Physica Medica 1997;XIII:187-94.

[12] Euratom basic safety standard directive-version 24 February 2010; S. Mundigl, revision of the euratom basic safety standards directive - current status. Radiation Protection Dosimetry 2010 [special issue of 19.10.2010].

[13] - http://www.ilo.org/public/english/bureau/stat/isco/docs/ gdstruct08.doc.

[14] OJ L 225. Council directive 2005/36/EC of the European parliament and of the council of 7 September 2005 on the recognition of professional qualifications; 30.9.2005. p. 22.

[15] Mills Michael D, Elson Howard R, Orton Colin G. "The terminal M.S. degree is no longer appropriate for students interested in a career in clinical medical physics in the United States", point/counterpoint. Medical Physics 2011;38(4):1737-9.

[16] Rogers David WO, Molloy Janelle A, Orton Colin G. "All graduate medical physics programs should have an original research component", point/counterpoint. Medical Physics 2011;38(5):2315-7.

[17] IAEA. Training course series no. 37, Clinical training of medical physicists specializing in radiation oncology. Vienna: IAEA; 2009.

[18] IAEA. Training course series no. 47, Clinical training of medical physicists specialising in diagnostic radiology. Vienna: IAEA; 2010.

[19] IAEA. Training course series no. 50, Clinical training of medical physicists specialising in nuclear medicine. Vienna: IAEA; 2011. 